\title{
MINIMIZATION OF NONSMOOTH INTEGRAL FUNCTIONALS
}

\author{
NIKOLAOS S. PAPAGEORGIOU \\ National Technical University \\ Department of Mathematics \\ Athens, 15773 Greece \\ APOSTOLOS S. PAPAGEORGIOU \\ R.P.I. \\ Department of Civil Engineering \\ Troy, New York 12180-3590 USA
}

(Received May 18, 1989 and in revised form February 28, 1990).

\begin{abstract}
In this paper we examine optimization problems involving multidimensional nonsmooth integral functionals defined on Sobolev spaces. We obtain necessary and sufficient conditions for optimality in convex, finite dimensional problems using techniques from convex analysis and in nonconvex, finite dimensional problems, using the subdifferential of Clarke. We also consider problems with infinite dimensional state space and we finally present two examples.
\end{abstract}

KEY WORDS AND PHRASES. Sobolev space, convex integrand, convex subdifferential, Clarke's subdifferential, $\epsilon$-subdifferential, Young-Fenchel equality.

1980 AMS SUBJECT CLASSIFICATION. 49A37.

\section{INTRODUCTION.}

The importance of the problem of minimization of a multidimensional integral functional defined on a Sobolev space, is well documented in the books of Ekeland-Temam [3] and Ladyzhenskaya-Uraltseva [6]. Various problems in calculus of variations, optimal control of distributed parameter systems and mechanics involve such a minimization.

In this note we obtain some necessary and sufficient conditions for the existence of a minimum or $\epsilon$-minimum of an integral functional defined on a Sobolev space. However contrary to most of the works in the literature, we consider nonsmooth integrands. Using concepts and techniques form nonsmooth analysis, we are able to obtain necessary and sufficient conditions for optimality in finite dimensional, convex problems (see theorem 3.1 and corollary I), in finite dimensional nonconvex problems (see theorem 4.1) and in infinite dimensional problems (see theorem 5.1). Finally we present two examples illustrating the applicability of our results.

2. PRELIMINARIES.

In this section we briefly recall some of the basic notions and facts from nonsmooth analysis that we will need in the sequel. For more details we refer to the works of Clarke [2], 
Ekeland-Temam [3] and Rockafellar [7].

Let $X$ be a real normed space and $X^{*}$ its dual. consider any function $f: X \rightarrow \overline{\mathbb{R}}=\mathbb{R} \cup\{+\infty\}$. The conjugate of $f(\cdot)$ is the function $f^{*}: X^{*} \rightarrow \overline{\mathbb{R}}$ defined by $f^{*}\left(x^{*}\right)=\sup \left\{\left(x^{*}, x\right)-f(x): x \in X\right\}$. Here $(\cdot, \cdot)$ denotes the duality brackets for the pair $\left(X, X^{*}\right)$. It is clear from this definition that for all $x^{*} \in X^{*}$, we have $\left(x^{*}, x\right) \leq f^{*}\left(x^{*}\right)+f(x)$. This inequality is known as the "Young-Fenchel inequality". Given a proper convex function $f: X \rightarrow \overline{\mathbb{R}}$ (proper meaning that $f(\cdot)$ is not identically $+\infty$ ), the convex subdifferential of $f(\cdot)$, is the generally multivalued mapping $\partial f: X \rightarrow X^{*}$ defined by $\partial f(x)=\left\{x^{*} \in X^{*}:\left(x^{*}, y-x\right) \leq\right.$ $f(y)-f(x)$ for all $y \in X\}$. The elements of $\partial f(x)$ are called subgradients of $f(\cdot)$ at $x$. It is clear that $\partial f(x)$ is always a closed, convex, maybe empty subset of $X^{*}$. Let $C$ be a closed convex subset of $X$ and let $\delta_{C}(\cdot)$ be its indicator function; i.e. $\delta_{C}(x)=0$ if $x \in C,+\infty$ otherwise. Then $\partial \delta_{C}(x) \neq \emptyset$ î and only if $x \in C$ and $\partial \delta_{C}(x)=N_{C}(x)=\left\{x^{*} \in X^{*}:\left(x^{*}, y-x\right) \leq 0\right.$ for all $y \in C$ \}, the normal cone to $C$ at $x$. If $C$ is an affine space parallel to a subspace $V$, then $N_{C}(x)=V^{\perp}$. If $f(\cdot)$ is Gateaux differentiable at $x$, then $\partial f(x)=\{\nabla f(x)\}$. Also using the subdifferential, we can have the following generalization of a well known optimality condition concerning the minimum of $f(\cdot)$. So for a proper, convex function $f(\cdot)$, the minimum (global) of $f(\cdot)$ over $X$ is attained at $x \in X$ if and only if $0 \in \partial f(x)$. Also it is easy to check that $x^{*} \in \partial f(x)$ if and only if $f(x)+f^{*}\left(x^{*}\right)=\left(x^{*}, x\right)$ ("Young-Fenchel equality"). More generally, given any $\epsilon \geq 0$ and a proper convex function $f(\cdot)$, the $\epsilon$-subdifferential of $f(\cdot)$ at $x$ is defined by $\partial_{\epsilon} f(x)=\left\{x^{*} \in X^{*}:\left(x^{*}, y-x\right)-\epsilon \leq f(y)-f(x)\right.$ for all $\left.y \in X\right\}$. Clearly if $\epsilon=0$, we recover the convex subdifferential defined above. If $f \in \Gamma_{0}(X)=$ \{proper, lower semicontinuous, convex functions $\}$ and $x \in \operatorname{dom} f=\{z \in X: f(z)<\infty\}$, then $\partial_{\epsilon} f(x) \neq \emptyset$ for $\epsilon>0$. Dually we can define $\partial_{\epsilon} f(x)$ by saying that $x^{*} \in \partial_{\epsilon} f(x)$ if and only if $f(x)+f^{*}\left(x^{*}\right)-\left(x^{*}, x\right) \leq \epsilon$. Again $\partial_{\epsilon} \delta_{C}(x)=N_{C}^{\epsilon}(x)=\left\{x^{*} \in X^{*}:\left(x^{*}, y-x\right) \leq \epsilon\right.$ for all $\left.y \in C\right\}$, the set of $\epsilon$-normals to $C$ at $x$. Note that $N_{C}^{\epsilon}(x), \epsilon>0$ is no longer a cone.

Let $f: X \rightarrow \mathbb{R}$ be a locally Lipschitz function. Following Clarke [2], we define $f^{0}(x ; h)=\varlimsup_{\substack{y \rightarrow x \\ \lambda ! 0}} \frac{f(y+\lambda h)-f(y)}{\lambda}$, the generalized directional derivative of $f(\cdot)$ at $x$ in the direction $h$. It is easy to check that $f^{0}(x ; \cdot)$ is finite, positively homogeneous, subadditive and satisfies $\left|f^{0}(x ; h)\right| \leq k\|h\|$. So we can define the set

$$
\partial_{c} f(x)=\left(x^{*} \in X^{*}:\left(x^{*}, h\right) \leq f^{0}(x ; h) \text { for all } h \in \dot{X}\right\} .
$$

We call $\partial_{c} f(x)$ the Clarke (or generalized) subdifferential of $f(\cdot)$ at $x \in X$. If $f(\cdot)$ is also convex, then the Clarke and convex subdifferentials coincide; i.e. $\partial f(x)=\partial_{c} f(x)$.

Let $f^{\prime}(x ; h)=\lim _{\lambda \perp 0} \frac{f(x+\lambda h)-f(x)}{\lambda}$. If $f^{\prime}(x ; h)$ exists for all $h \in X$ and $f^{\prime}(x ; h)=f^{0}(x ; h)$, $h \in X$, then $f(\cdot)$ is said to be regular at $x$. This is a fairly large class of functions that includes the convex continuous functions and the functions that are strictly differentiable at $x$.

\section{CONVEX INTEGRAND.}

Let $Z$ be a bounded domain in $\mathbb{R}^{n}$ with smooth boundary $\partial Z=\Gamma$. We will be studying the following optimization problem, with $\phi \in W^{1, p}(Z), 1<p<\infty$

$$
\inf \left\{\int_{Z} L(z, x(z), \nabla x(z)) d z: x \in \phi+W_{0}^{1, p}(Z)\right\} \text {. }
$$

In this section we consider the case where the integrand $L(\cdot, \cdot, \cdot)$ is convex in the last 
two variables. We will need the following hypothesis:

$H(L): \quad L: Z \times \mathbb{R} \times \mathbb{R}^{n} \rightarrow \mathbb{R}$ is an integrand s.t.

(1) $z \rightarrow L(z, x, y)$ is measurable,

(2) $(x, y) \rightarrow L(z, x, y)$ is continuous, convex,

(3) $|L(z, x, y)| \leq a(z)+b\left(|x|^{p}+\|y\|^{p}\right)$ a.e. with $a(\cdot) \in L_{+}^{1}$.

We have the following necessary and sufficient condition for optimality in problem (*).

THEOREM 3.1. If hypothesis $H(L)$ holds, then $x \in V=\phi+W_{0}^{1, p}(Z)$ solves $(*)$ if and only if there exists $v^{*} \in L_{n}^{q}(Z)=L^{q}\left(Z, \mathbf{R}^{n}\right) \quad$ with $\quad \operatorname{div}^{*} \in L^{q}(Z) \quad$ s.t. $\quad \operatorname{divv}^{*}(z) x(z)+$ $\left\langle v^{*}(z), \nabla x(z)\right\rangle=L(z, x(z), \nabla x(z))+L^{*}\left(z, \operatorname{div} v^{*}(z), v^{*}(z)\right)$ a.e. where $\langle\cdot, \cdot\rangle$ denote the inner product in $\mathbb{R}^{\mathbf{n}}$.

PROOF. Let $A: W^{1, p}(Z) \rightarrow L^{p}(Z) \times L_{n}^{p}(Z)$ be defined by $A x=(x, \nabla x)$. Clearly $A(\cdot)$ is linear, continuous. Furthermore we know (see for example Ekeland-Temam [3]), that $A^{*}: L^{q}(Z) \times L_{n}^{q}(Z) \rightarrow\left[W^{1, p}(Z)\right]^{*}(1 / p+1 / q=1)$ is defined by $A^{*}(x, y)=x-$ divy. Let $J_{L}$ : $L^{p}(Z) \times L_{n}^{p}(Z) \rightarrow \mathbb{R}$ be the integral functional defined by $J_{L}(x, y)=\int_{Z} L(z, x(z), y(z)) d z$. Then problem (*) takes the following equivalent form:

$$
\inf \left\{\left(J_{L} o A\right)(x): x \in \phi+W_{0}^{1, p}(Z)=V\right\}
$$

Since the cost functional is convex (hypothesis $H(L)(2)$ ), from the convex analysis (see section 2), we know that $x(\cdot) \in V$ solves problem (*)' (and so the equivalent problem (*)) if and only if $0 \in \partial\left(J_{L} o A\right)(x)+N_{V}(x)$. But $V$ is an affine space parallel to $W_{0}^{1, p}(Z)$. So $N_{V}(x)=W_{0}^{1, p}(Z)^{\perp}=\{0\}$. Hence $0 \in \partial\left(J_{L} o A\right)(x)$. Because of hypothesis $H(L), J_{L}(\cdot, \cdot)$ is continuous on $L^{p}(Z) \times L_{n}^{p}(Z)$. Therefore theorem 2, p. 201 of Ioffe-Tichomirov [5], tells us that $\partial\left(J_{L} o A\right)(x)=A^{*} \partial J_{L}(A x)$. From Rockafellar [7], we know that $\partial J_{L}(A x)=S_{\partial L\left(\cdot, x(\cdot), \nabla_{x(\cdot))}^{q}\right.}$ $=\left\{x^{*}(\cdot) \in L^{q}(Z) \times L_{n}^{q}(Z): x^{*}(z) \in \partial L(z, x(z), \nabla x(z)) a . e.\right\} \quad$ (here $\partial L(z, x, y)$ is the convex subdifferential of $L(z, \cdot, \cdot))$. So we have that $0 \in A^{*} \partial J_{L}(Z x)$ if and only if there exist $\left(w^{*}, v^{*}\right) \in L^{q}(Z) \times L_{n}^{q}(Z)$ s.t. $\left(w^{*}, v^{*}\right) \in \partial J_{L}(A x)$ and $A^{*}\left(w^{*}, v^{*}\right)=0$. From this last equation we get that $w^{*}-d i v v^{*}=0 \Rightarrow w^{*}=d i v v^{*}$. Then since $\left(\operatorname{div} v^{*}, v^{*}\right) \in \partial J_{L}(A x)=S_{\partial L\left(\cdot, x(\cdot), \nabla_{x(\cdot)}^{q}\right)}$, $\left(\operatorname{div} v^{*}(z), v^{*}(z)\right) \in \partial L(z, x(z), \nabla x(z))$ a.e. Invoking the Young-Fenchel equality, we get that

$$
\left.\operatorname{div}^{*}(z) . x(z)+\left\langle v^{*}(z), \nabla x(z)\right)\right\rangle=L(z, x(z), \nabla x(z))+L^{*}\left(z, \operatorname{div} v^{*}(z), v^{*}(z)\right) \text { a.e. }
$$

Q.E.D.

Using partial conjugates of $L(z, \cdot, \cdot)$ with respect to $x$ and $y$ respectively, we can have the following necessary condition for optimality concerning $(*)$. By $L^{*} 1$ (resp. $L^{*}$ ) we will denote the conjugate of $L(z, \cdot, y)$ (resp. of $L(z, x, \cdot)$ ).

COROLLARY I. If hypothesis $H(L)$ holds and $x \in V=\phi+W_{0}^{1, p}(Z)$ solves $(*)$, then there exists $v^{*}(\cdot) \in L_{n}^{q}(Z) \quad$ s.t. $\quad$ div $\quad v^{*}(\cdot) \in L^{q}(Z) \quad$ and $L(z, x(z), \nabla x(z))+L^{* 1}(z, d i v$ $\left.v^{*}(z), \nabla x(z)\right)=\operatorname{div} v^{*}(z) . x(z)$ a.e., $L(z, x(z), \nabla x(z))+L^{*}\left(z, x(z), v^{*}(z)\right)=\left\langle v^{*}(z), \nabla x(z)\right\rangle$ a.e.

PROOF. As in the proof of theorem 3.1, we get $v^{*}(\cdot) \in L_{n}^{q}(Z)$ s.t. $\operatorname{div} v^{*}(\cdot) \in L^{q}(Z)$ and $\left(\operatorname{div} v^{*}(z), v^{*}(z)\right) \in \partial L(z, x(z), \nabla x(z))$ a.e.

Applying proposition 2.3.15, p. 48 of Clarke [2] to $\partial L$ and using the Young-Fenchel equality, we get the corollary. 


\section{NONCONVEX INTEGRAND.}

In this section we drop the convexity hypothesis on $L(z, \cdot, \cdot)$ and instead we assume that $L(z, \cdot, \cdot)$ is Lipschitz. Using Clarke's subdifferential, we derive a necessary condition for optimality in this nonconvex problem.

So our hypothesis about $L(\cdot, \cdot, \cdot)$ is now the following:

$H(L)^{\prime}: \quad L: Z \times \mathbb{R} \times \mathbb{R}^{\boldsymbol{n}} \rightarrow \mathbb{R}$ is an integrand s.t.

(1) $z \rightarrow L(z, x, y)$ is measurable,

(2) for all $(x, y),\left(x^{\prime}, y^{\prime}\right) \in \mathbf{R} \times \mathbf{R}^{n}$, we have $\left|L(z, x, y)-L\left(z, x^{\prime}, y^{\prime}\right)\right| \leq k(z)$ $\left(\left|x-x^{\prime}\right|+\left\|y-y^{\prime}\right\|\right)$ a.e. with $k(\cdot) \in L_{+}^{q}(Z)$

THEOREM 4.1. If hypothesis $H(L)^{\prime}$ holds and $x \in V=\phi+W_{0}^{1, p}(Z)$ solves $(*)$, then there exists $v^{*}(\cdot) \in L_{n}^{q}(Z)$ s.t. $d i v v^{*}(\cdot) \in L^{q}(Z)$ and $\left(\operatorname{div} v^{*}(\cdot), v^{*}(\cdot)\right) \in \partial_{c} L(z, x(z), \nabla x(z))$ a.e.

PROOF. Using the corollary on page 52 of Clarke [2], we know that since $x \in V$ solves (*), then $0 \in \partial_{c}\left(J_{L} o A\right)(x)+N_{V}(x)=\partial_{c}\left(J_{L} o A\right)(x)$ (since $N_{V}(x)=W_{0}^{1, p}(Z)^{\perp}=\{0\}$ ). From proposition 2, p. 216 of Aubin [1], we know that $\partial_{c}\left(J_{L} o A\right)(x) \subseteq A^{*} \partial_{c} J_{L}(A x)$, while from

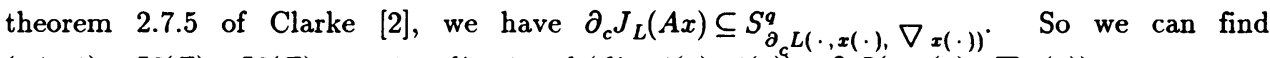
$\left(w^{*}, v^{*}\right) \in L^{q}(Z) \times L_{n}^{q}(Z)$ s.t. $w^{*}=\operatorname{div} v^{*}$ and $\left(\operatorname{div} v^{*}(z), v^{*}(z)\right) \in \partial_{c} L(z, x(z), \nabla x(z))$ a.e.

$\underline{\underline{Q . E . D .}}$

REMARKS. (1) If $L(z, \cdot, \cdot)$ is regular at $(x(z), \nabla x(z))$ for almost all $z \in Z$, then using proposition 2.3.15, p. 48 of Clarke [2], we can also say that $\operatorname{div} v^{*}(z) \in \partial_{c 1} L(z, x(z), \nabla x(z))$ a.e. and $v^{*}(z) \in \partial_{c 2} L(z, x(z), \nabla x(z))$ a.e., where $\partial_{c 1} L$ denotes the Clarke subdifferential of $L(z, \cdot, y)$ and $\partial_{c 2} L(z, x, \cdot)$ the Clarke subdifferential of $L(z, x, \cdot)$.

(2) If $L(z, \cdot, \cdot)$ is a $C^{1}$-function at $(x(z), \nabla x(z))$ for almost all $z \in Z$, then theorem 4.1 combined with remark $(\underline{1})$ above tells us that there exists $v^{*}(\cdot) \in L_{n}^{q}(Z)$ s.t. $\operatorname{div} v^{*}(\cdot) \in L^{q}(Z)$, $\operatorname{div} v^{*}(z)=L_{x}^{\prime}(z, x(z), \nabla x(z))$ a.e. and $v^{*}(z)=L_{y}^{\prime}(z, x(z), \nabla x(z))$ a.e.

5. INFINITE DIMENSIONAL EVOLUTIONS.

In this section $n=1$ (time variable) and the state space is infinite dimensional. So let $T=[0, b]$ and $X$ be a separable, reflexive Banach space. We consider the following optimization problem:

$$
m=\inf \left\{\int_{0}^{b} L(t, \dot{x}(t)) d t: x(0)=x_{0}, x(b)=k^{\prime}, x(\cdot) \in W^{i, p}(T, X)\right\}
$$

Here $W^{1, p}(T, X)$ is the space of all $X$-valued distributions $x(\cdot)$ s.t. $\dot{x} \in L^{p}(X)$ the derivative taken in the sense of distributions. Recall that $W^{1, p}(T, X)$ is a Banach space with the norm $\|x\|_{W^{1, p}}=\left[\|x\|_{L^{p}(X)}^{p}+\|\dot{x}\|_{L^{p}(X)}^{p}\right]^{1 / p}$. Also it is well known that each function in $W^{1, p}(T, X)$ is almost everywhere equal to an absolutely continuous function and so the boundary conditions in $(* *)$ make sense.

Let $\eta: W^{1, p}(T, X) \rightarrow L^{p}(T, X)$ be defined by $\eta(x)=\dot{x}$ and consider $V_{1}=\left\{x \in W^{1,0}(T, X)\right.$ : $\left.x(0)=x_{0}, x(b)=k^{\prime}\right\}$, and $V_{2}=\left\{v \in L^{p}(X): \int_{0}^{b} v(s) d s=k^{\prime}-x_{0}=k\right\}$. Then $\left.\eta\right|_{v_{1}}$ a bijection from $V_{1}$ to $V_{2}$ and so we deduce that (**) is equivalent to the following optimization problem:

$$
\inf \left\{\int_{0}^{b} L(t, y(t)) d t: y \in L^{p}(X), \int_{0}^{b} y(s) d s=k^{\prime}-x_{0}=k\right\}
$$


Note that $(* *)^{\prime}$ is equal to $\left(\oint_{0}^{b} L_{t}\right)(k)$, where $\left(\oint_{0}^{b} L_{t}\right)(\cdot)$ is the continuous infimal convolution introduced by Ioffe-Tichomirov [5] (section 8.3.2).

We have the following necessary and sufficient condition for (approximate) optimality in $(* *)^{\prime}$ and hence in the equivalent problem $(* *)$.

THEOREM 5.1. If $L: T \times X \rightarrow \overline{\mathbb{R}}$ is a measurable integrand, convex in $x$ and assume that $\partial\left(\oint_{0}^{b} L_{t}\right)(k) \neq \phi$, then $x(\cdot) \in W^{1, p}(T, X)$ is $\epsilon$-optimal for $(* *) \epsilon \geq 0$, if and only if there exists $\quad x^{*} \in X^{*} \quad$ and $\quad \epsilon(\cdot) \in L_{+}^{1} \quad$ s.t. $\quad x^{*} \in \partial_{\epsilon(t)} L(t, \dot{x}(t)) \quad$ a.e., $\quad \int_{0}^{b} \epsilon(s) d s \leq \epsilon \quad$ and $\int_{0}^{b} \dot{x}(s) d s=k$.

\section{PROOF. Sufficiency}

From the definition of the $\epsilon$-differential (see section 2), we know that for any $y(\cdot) \in L^{1}(Z)$ we have:

$$
\begin{gathered}
\left(x^{*}, y(t)-\dot{x}(t)\right)-\epsilon(t) \leq L(t, y(t))-L(t, \dot{x}(t)) \text { a.e. } \\
\Rightarrow \int_{0}^{b} L(t, \dot{x}(t)) d t+\left(x^{*}, \int_{0}^{b}(y(t)-\dot{x}(t)) d t\right)-\int_{0}^{b} \epsilon(t) d t \leq \int_{0}^{b} L(t, y(t)) d t .
\end{gathered}
$$

Let $\mathcal{L}^{1}(k)$ be the subset of $L^{1}(Z)$ defined by

$$
\mathcal{L}^{1}(k)=\left\{y(\cdot) \in L^{1}(X): \int_{0}^{b} y(t) d t=k\right\} .
$$

Taking the infimum over $\mathcal{L}^{1}(k)$ of both sides of the last inequality, we get

$$
\int_{0}^{b} L(t, \dot{x}(t)) d t-\int_{0}^{b} \epsilon(t) d t \leq\left[\oint_{0}^{b} L_{t} d t\right](k)=m .
$$

But $\int_{0}^{b} \epsilon(t) d t \leq \epsilon$. So we have that

$$
\int_{0}^{b} L(t, \dot{x}(t)) d t \leq m+\epsilon .
$$

Necessity:

Let $x^{*} \in \partial\left[\oint_{0}^{b} L_{t} d t\right](k)$. Consider the function $u(t, y)=L(t, \dot{x}(t))-L(t, y)+\left(x^{*}, y-x^{*}(t)\right)$ and set $\epsilon(t)=\sup _{y \in X} u(t, y)$. By taking $y=\dot{x}(t)$ for every $t \in T$, we can see that $\epsilon(t) \geq 0$. Also observe that given $\lambda \geq 0, \epsilon(t)>\lambda$ if and only if there exists $y \in X$ s.t. $u(t, y)>\lambda$. Hence $\{t \in T: \epsilon(t)>\lambda\}=\operatorname{proj}_{T}\{(t, y): u(t, y)>\lambda\}$. Since $u(\cdot, \cdot)$ is measurable, the von Neumann projection theorem (see Saint-Beuve [8]) tells us that $\operatorname{proj}_{T}\{(t, y): u(t, y)>\lambda\}$ is a Lebesgue measurable subset of $T$. Hence $\epsilon(\cdot)$ is Lebesgue measurable.

For all $(t, y) \in T \times X$, we have that

$$
\begin{gathered}
L(t, \dot{x}(t))-L(t, y)+\left(x^{*}, y-\dot{x}(t)\right) \leq \epsilon(t) \\
\Rightarrow x^{*} \in \partial_{\epsilon(t)} L(t, \dot{x}(t)) \text { for all } t \in T .
\end{gathered}
$$


We claim that $\int_{0}^{b} \epsilon(t) d t \leq \epsilon$. To this end, observe that since $x^{*} \in \partial\left[\oint_{0}^{b} L_{t} d t\right](k)$, for all $y \in X$, we have

$$
\left(\oint_{0}^{b} L_{t} d t\right)(y)-\left(\oint_{0}^{b} L_{t} d t\right)(k) \geq\left(x^{*}, y-k\right) .
$$

So for all $y(\cdot) \in L^{1}(X)$, we have:

$$
\begin{gathered}
\left(x^{*}, \int_{0}^{b}(y(t)-\dot{x}(t)) d t\right) \leq \int_{0}^{b} L(t, y(t)) d t-\int_{0}^{b} L(t, \dot{x}(t)) d t+\epsilon \\
\quad \Rightarrow \int_{0}^{b}\left(L(t, \dot{x}(t))-L(t, y(t))+\left(x^{*}, y(t)-\dot{x}(t)\right) d t \leq \epsilon\right. \\
\Rightarrow \epsilon \geq \sup \left[\int_{0}^{b}\left(L(t, \dot{x}(t))-L(t, y(t))+\left(x^{*}, y(t)\right)\right) d t: y(\cdot) \in L^{1}(X)\right] \\
\quad=\int_{0}^{b} \sup \left[L(t, \dot{x}(t))-L(t, y)+\left(x^{*}, y-\dot{x}(t)\right): y \in X\right] d t
\end{gathered}
$$

(see theorem 2.2 of Hiai-Umegaki [4]),

$$
=\int_{0}^{b} \epsilon(t) d t
$$

6. EXAMPLES.

Q.E.D.

(i) Consider the following minimization problem:

$$
\inf \left\{J(x)-\int_{0}^{1} t \dot{x}(t)^{2} d t: x(\cdot) \in W^{1,2}(T), x(0)=1, x(1)=0\right\}=m_{1} .
$$

Let $x_{k}(t)=1$ for $0 \leq t \leq k^{-1}$ and $x_{k}(t)=\ln t / \ln k$ for $k^{-1} \leq t \leq 1$. We have $J\left(x_{k}\right)=(\ln k)^{-1} \rightarrow 0$ as $k \rightarrow \infty$. So $m_{1}=0$. But this value of $(* * *)_{1}$ is not realized by any $x(\cdot) \in W^{1,2}(T)$, because then $t \dot{x}(t)^{2}=0$ a.e. $\Rightarrow \dot{x}(t)=0$ a.e. $\Rightarrow x(\cdot)$ is a constant, a violation of the boundary conditions. Another minimizing sequence for $(* * *)_{1}$ is $x_{n}(t)=t^{\frac{1}{n}} t \in[0,1]$.

So we can only find $\epsilon$-optimal solutions for $(* * *)_{1}$. According to theorem 5.1, $x(\cdot) \in W^{1,2}(T)$ is an $\epsilon$-optimal solution of $(* * *)_{1}$ if and only if there exists $x^{*} \in \mathbb{R}$ and $\epsilon(\cdot) \in \mathcal{L}^{1}(\epsilon)$ s.t. $(\alpha) \int_{0}^{1} \epsilon(s) d s \leq \epsilon,(\beta) x^{*} \in \partial_{\epsilon(s)}\left(s \dot{x}(s)^{2}\right)=[2(s \dot{x}(s)-\sqrt{s \in(s))}, 2(s \dot{x}(s)+\sqrt{s \in(s))}]$ a.e. and $(\gamma) \int_{0}^{1} \dot{x}(s) d s \stackrel{0}{=}-1$.

So let $\epsilon(t)=\epsilon$. From $(\alpha),(\beta)$ and $(\gamma)$ above, we deduce that $x^{*}=0$ satisfies $(\gamma)$ if and only if $-\sqrt{\epsilon} \leq \sqrt{t} \dot{x}(t) \leq \sqrt{\epsilon}, t \in[0,1]$. Thus we have that $\dot{x}_{n}(t)=t^{\frac{1}{n}-1} / n t \in[0,1], n \geq 1$ is the derivative of an $\epsilon$-optimal solution if $n \geq \frac{1}{\sqrt{\epsilon}}$. Therefore $x_{n}(t)=t^{1 / n}$ is an $\epsilon$-optimal solution if $n \geq \frac{1}{\sqrt{\epsilon}}$. So we have produced the second of the minimizing sequences mentioned above.

(ii) Let $Z$ be a bounded domain in $\mathbb{R}^{n}$ with smooth boundary. We consider "Plateau's problems":

$$
\inf \left\{J(x)=\int_{Z}\left(1+\|\nabla x(z)\|^{2}\right)^{1 / 2} d z: x \in \phi+W_{0}^{1,2}(Z)=V\right\}
$$


where $\phi \in W^{1,2}(Z)$.

Invoking theorem 3.1 of this paper (special case where the integrand is independent of $(z, x)$ ), we have that $x(\cdot) \in V$ is a solution of $(* * *)_{2}$ if and only if there exists $v^{*}(\cdot) \in L^{2}(Z)$ s.t. $\operatorname{div} v^{*}=0 \quad$ and $\quad\left(\right.$ i) $\quad\left\|v^{*}(z)\right\| \leq 1 \quad$ a.e. $\quad$ (ii) $\quad\left(1+\|\nabla x(z)\|^{2}\right)^{1 / 2}-\left(1-\left\|v^{*}(z)\right\|^{2}\right)^{1 / 2}=$ $\left\langle\nabla x(z), x^{*}(z)\right\rangle$ a.e.

If we express those optimality conditions in terms of the subdifferential of the cost integrand $\left(1+\|y\|^{2}\right)^{1 / 2}$, we have

$$
v^{*}(\cdot)=\partial J(x)=\nabla J(x)=\left\{\frac{\nabla x}{\left(1+\|\nabla x\|^{2}\right)^{1 / 2}}\right\}
$$

and so clearly $d i v v^{*}=0$. These are the optimality conditions obtained by Ekeland-Temam [3], chapter $\mathrm{V}$, section 1.1 and chapter $\mathrm{X}$, section 4.2 .

ACKNOWLEDGEMENT. The authors are indebted to the referee for his (her) corrections, suggestions and remarks that improved the content of this paper considerable. The research of the first author was supported by N.S.F. Grant DMS- 8802688 .

\section{REFERENCES}

1. AUBIN, J.-P. Gradients genéralisés de Clarke, Annales Sci. Math. Quebec 2 (1978), pp. 197-252.

2. CLARKE, F.H. Optimization and Nonsmooth Analysis, Wiley, New York, (1983).

3. EKELAND, I. and TEMAM, R. Convex Analysis and Variational Problems, North Holland, Amsterdam, (1974).

4. HIAI, F. and UMEGAKI, H. Integrals conditional expectations and martingales of multivalued functions, J. Multiv. Anal. I (1977), pp. 149-182.

5. IOFFE, A. and TICHOMIROV, V. Theory of Extremal Problems, North Holland, Amsterdam, (1978).

6. LADYZHENSKAYA, $O$. and URALTSEVA, $N$. Linear and Quasilinear Elliptic Equations, Academic Press, New York, (1968).

7. ROCKAFELLAR, R.T. Conjugate Duality and Optimization, Conference Board of Mathematical Sciences Series, No. 16, SIAM Publications, Philadelphia, (1974).

8. SAINT BEUVE, M.-F. On the extension of von Neumann-Aumann's theorem, J. Funct. Anal. 17, (1974), pp. 112-129. 


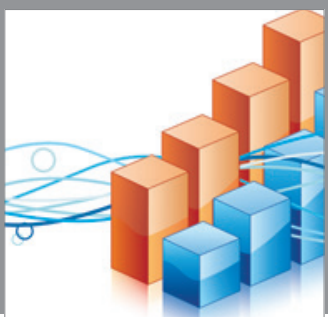

Advances in

Operations Research

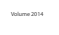

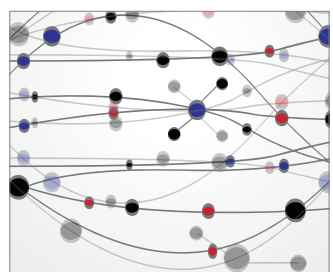

\section{The Scientific} World Journal
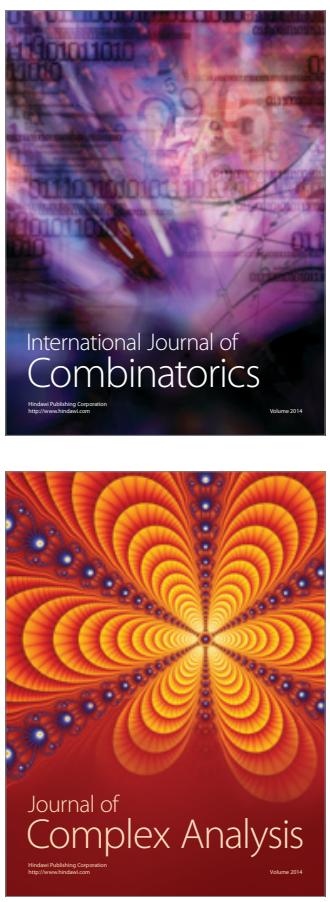

International Journal of

Mathematics and

Mathematical

Sciences
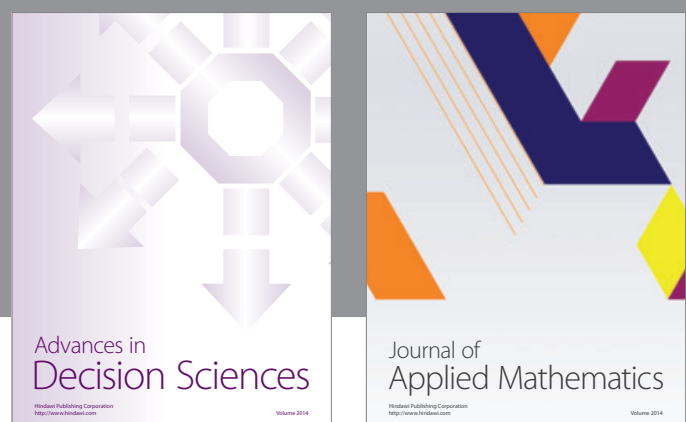

Journal of

Applied Mathematics
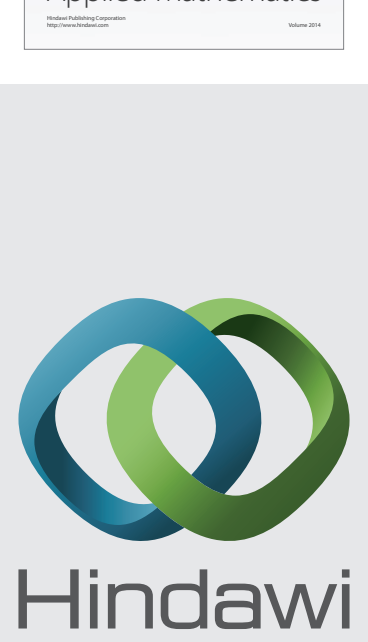

Submit your manuscripts at http://www.hindawi.com
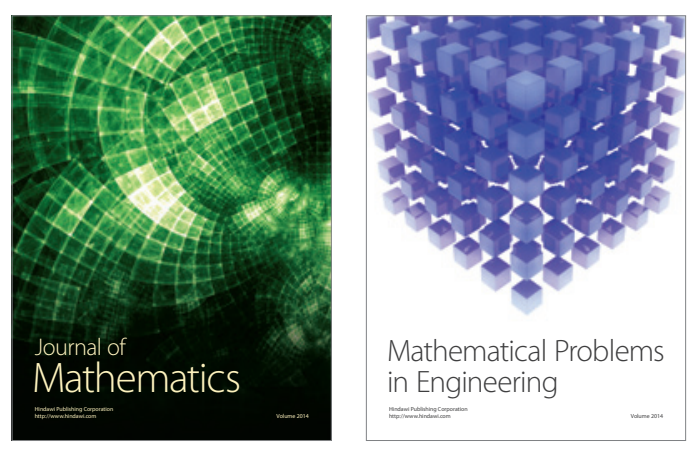

Mathematical Problems in Engineering
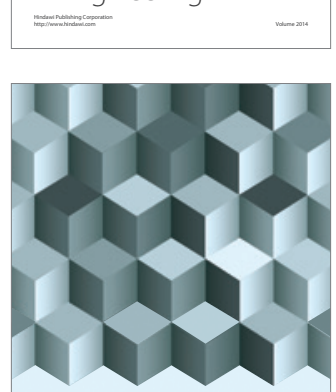

Journal of

Function Spaces
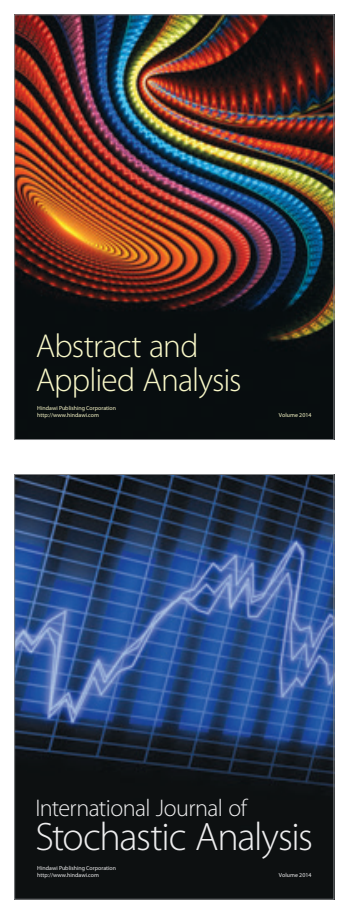

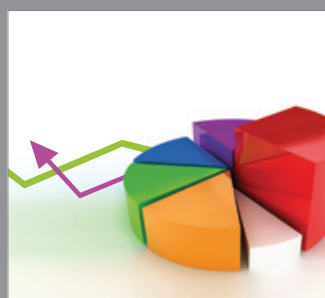

ournal of

Probability and Statistics

Promensencen
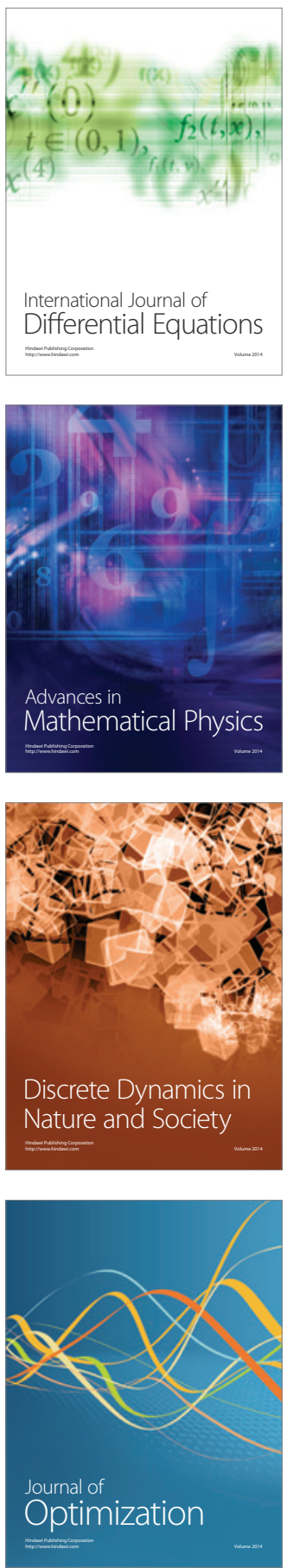